\title{
https://doi.org/10.46813/2021-134-106 \\ DIFFRACTION RADIATION OF PARTICLE PASSING \\ BY IMPEDANCE WEDGE
}

\section{V.M. Ostroushko \\ National Science Center “Kharkov Institute of Physics and Technology”, Kharkiv, Ukraine E-mail: ostroushko-v@kipt.kharkov.ua}

The diffraction radiation field generated by the charged particle passing by the perfectly conductive or impedance wedge is considered. With perfect conductivity, the wedge rotation around the edge fixed along with the straight particle motion line, does not change the total radiated energy. When the motion to the edge is almost parallel to a face of wedge then an increase of impedance from zero increases the radiated energy.

PACS: 41.60.-m

\section{INTRODUCTION}

When bunches of the charged particles formed in accelerator pass by metal or dielectric structures then a diffraction radiation is emitted. It may be wide-band [1], and the structure plays a role of antenna. To get effective emission in the wide wavelength range, it is preferably for the antenna to have no characteristic length. In the paper [2], the diffraction radiation is studied, which arises when the charged particle passes by perfectly conductive half-plane in the direction perpendicular to the edge of the half-plane. In the manual [3], the case of arbitrary direction is considered. In the present work, an impedance wedge plays a role of antenna, and the charged particle is moving uniformly along a line, which does not cross the wedge faces and is not parallel to any of them.

\section{PROBLEM FORMULATION}

In the following study, some frames of reference are used (Fig. 1). The Cartesian coordinates $(x, y, z)$, with the axis $z$ directed along the edge of wedge, are connected with the coordinates $r, \theta, \rho$, and $\varphi$, $z=r \cos \theta, \quad \rho=r \sin \theta, \quad x=\rho \cos \varphi, \quad y=\rho \sin \varphi$, $\theta \in(0, \pi)$, and the sector $\varphi \in(-\Phi, \Phi)$, with $\Phi \in(\pi / 2, \pi)$, is free space. In the Cartesian coordinates $(\xi, \eta, \zeta)$, the axis $\zeta$ is the line of particle's motion, the axis $\xi$ is directed to the edge of wedge along the shortest line segment between two points, at the motion line and at the edge, and $\xi_{\mathrm{e}}$ denotes the segment length. In the planes perpendicular to the edge of wedge, the Cartesian coordinate system $\left(x^{\prime}, y^{\prime}\right)$ has an origin at the edge and the axis $x^{\prime}$ is parallel to the projection of the motion line on the planes. The angle between the directions of the axes $\zeta$ and $z$ is denoted $\theta_{\mathrm{e}}, \theta_{\mathrm{e}} \in(0, \pi)$, and the angle between the axes $x$ and $x^{\prime}$ is denoted $\varphi_{\mathrm{e}}$. It is assumed that the particle moves with $y$ value increase, and so, the wedge is in the half-space $y^{\prime} \geq 0$, and $\varphi_{\mathrm{e}} \in(\pi-\Phi, \Phi)$. The defined coordinates are connected, $\quad z=\zeta \cos \theta_{\mathrm{e}}+\eta \sin \theta_{\mathrm{e}}, \quad x^{\prime}=\zeta \sin \theta_{\mathrm{e}}-\eta \cos \theta_{\mathrm{e}}$, $y^{\prime}=\xi-\xi_{\mathrm{e}}, x^{\prime}=\rho \cos \left(\varphi-\varphi_{\mathrm{e}}\right), y^{\prime}=\rho \sin \left(\varphi-\varphi_{\mathrm{e}}\right)$.
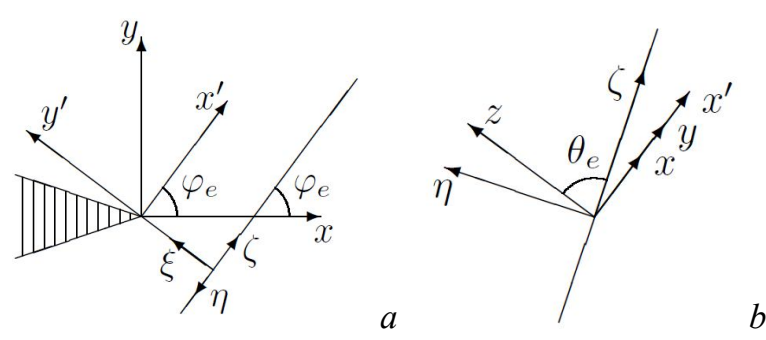

Fig. 1. The views from $z=+\infty$ (a) and $\xi=-\infty$ (b)

It is considered the radiation caused by the straightline and uniform motion of the particle, so that $\xi=0$, $\eta=0, \zeta=\beta c t$, where $t$ is time, $c$ is the speed of light, $\beta \in(0,1)$. In free space, the electromagnetic field obeys Maxwell's equations, and it may be given by the sum of E- and H-waves. The space-time dependence of electric and magnetic field strength components of these waves has the form $\operatorname{Re}\left[\vec{F}(x, y) \exp \left(i k_{z} z-i \omega t\right)\right]$, where $F$ stands for $E$ or $H$. The amplitudes of the longitudinal components obey the equations $\nabla_{x y}^{2} F_{z}+k_{x y}^{2} F_{z}=0$, where $\nabla_{x y}=\vec{e}_{x} \partial_{x}+\vec{e}_{y} \partial_{y}, k_{x y}=\left(k^{2}-k_{z}^{2}\right)^{1 / 2}, k=\omega / c, \vec{e}$ with index is unit vector in relevant direction, and $\partial$ with index is derivative with respect to relevant variable. The transverse component amplitudes are determined by the longitudinal ones,

$$
\begin{aligned}
& k_{x y}^{2} \vec{E}_{x y}=+i k\left[\nabla H_{z} \vec{e}_{z}\right]+i k_{z} \nabla_{x y} E_{z}, \\
& k_{x y}^{2} \vec{H}_{x y}=-i k\left[\nabla E_{z} \vec{e}_{z}\right]+i k_{z} \nabla_{x y} H_{z} .
\end{aligned}
$$

It is assumed that impedance $\mathrm{Z}$ for both faces is identical and depends on frequency, $Z=\left(-i k \delta_{\mathrm{s}}\right)^{\sigma}$, where $\delta_{\mathrm{s}}$ is skin layer depth and the power index $\varpi$ is connected with the skin effect type. That is, $\mathrm{Z}=\left(k \delta_{\mathrm{s}}\right)^{\varpi} \exp \left(-i \alpha_{\mathrm{s}}\right)$, where $\alpha_{\mathrm{s}}=\varpi \pi / 2$. If one proceeds from the equality $Z=\left\{1-\omega_{\mathrm{e}}^{2}\left[\omega\left(\omega+i \nu_{\mathrm{c}}\right)\right]^{-1}\right\}^{-1 / 2}$, where $\omega_{\mathrm{e}}$ and $v_{\mathrm{c}}$ are plasma and collision frequencies, then the cases of normal and anomalous skin effect (related to the frequency ranges $\omega<<v_{\mathrm{c}}<<\omega_{\mathrm{e}}$ and $\left.v_{\mathrm{c}}<<\omega<<\omega_{\mathrm{e}}\right)$ correspond to $\left\{\varpi=1 / 2, \delta_{\mathrm{s}}=c v_{\mathrm{c}} / \omega_{\mathrm{e}}^{2}\right\}$ and $\left\{\varpi=1, \delta_{\mathrm{s}}=c / \omega_{\mathrm{e}}\right\}$, respectively, and dependence of $\mathrm{Z}$ on $k$ is near to power one at $k \delta_{\mathrm{s}}<<1$ (and then $|\mathrm{Z}|<<1)$.

Both for E-waves, and for H-waves the problem is reduced to the plane one: for the given field of an exter- 
nal source $F_{z}^{\mathrm{e}}$, it should be found the scattered field $F_{z}^{\mathrm{s}}$, which should obeys the equation $\nabla_{x y}^{2} F_{z}^{\mathrm{s}}+k_{x y}^{2} F_{z}^{\mathrm{s}}=0$, inside the sector $\varphi \in(-\Phi, \Phi)$, the radiation condition, $\int_{-\Phi}^{+\Phi} d \varphi \rho\left|\partial_{\rho} F_{z}^{\mathrm{s}}-i k_{x y} F_{z}^{\mathrm{s}}\right|^{2} \rightarrow 0$, at $\rho \rightarrow \infty$, the condition of the functions $\left|F_{z}^{\mathrm{s}}\right|^{2}$ and $\left|\nabla_{x y} F_{z}^{\mathrm{s}}\right|^{2}$ integrability over any bounded area (Meixner's condition), and the full field, $\vec{F}^{\mathrm{f}}=\vec{F}^{\mathrm{s}}+\vec{F}^{\mathrm{e}}$, should obey, at $\varphi= \pm \Phi$, the impedance boundary conditions, $\pm E_{z}^{\mathrm{f}} / H_{\rho}^{\mathrm{f}}=\mathrm{Z}=\mp E_{\rho}^{\mathrm{f}} / H_{z}^{\mathrm{f}}$, which, with taking (1) into account, may be written in the form

$$
\left(\rho F_{z}^{\mathrm{f}}\right)^{-1} \partial_{\varphi} F_{z}^{\mathrm{f}}= \pm i k_{x y} \sin \chi_{F},
$$

where $\chi_{E, H}$ are determined by the equations,

$$
\sin \chi_{E}=k_{x y} /(k Z), \sin \chi_{H}=\left(k_{x y} Z\right) / k,
$$

and the conditions $0<\operatorname{Re} \chi_{E, H} \leq \pi / 2$.

\section{ONE WAVE SCATTERING}

The solving of the problem of one plane wave scattering on impedance wedge is described circumstantially in the original papers and books $[4,5]$. The result is briefly described below. Let the incident scalar plane wave with unit amplitude in the 2D space has the spatial dependence $\exp \left(i k_{x}^{\prime} x^{\prime}+i k_{y}^{\prime} y^{\prime}\right)$, where $k_{x}^{\prime}$ is real and $k_{y}^{\prime}=\left(k_{x y}^{2}-k_{x}^{\prime 2}\right)^{1 / 2}$. Then the sum of the wave and the scattered field may be given in the form of Sommerfeld integral,

$$
\begin{gathered}
f_{z}(\chi, \rho, \varphi)=(2 \pi i)^{-1} \times \\
\times \int_{C} d w \exp \left(-i k_{x y} \rho \cos w\right) s_{z}(\chi, \varphi+w) .
\end{gathered}
$$

Here $s_{z}(\chi, w)=s_{0}(w) \Pi_{\Phi}(\chi, w) / \Pi_{\Phi}\left(\chi, w_{\mathrm{i}}\right)$, $s_{0}(w)=k_{\Phi} \cos \left(k_{\Phi} w_{\mathrm{i}}\right) /\left[\sin \left(k_{\Phi} w\right)-\sin \left(k_{\Phi} w_{\mathrm{i}}\right)\right]$,

$k_{\Phi}=\pi /(2 \Phi), \Pi_{\Phi}(\chi, w)=\prod_{\{ \pm\}} \psi_{\Phi / 2}((\pi / 2)-\chi \pm w)$,

$$
\begin{gathered}
\psi_{\Phi}(w)=\exp \left\{-\int_{0}^{\infty} d \tau[\cosh (\tau w)-1] \times\right. \\
\left.\times[2 \tau \cosh (\pi \tau / 2) \sinh (2 \Phi \tau)]^{-1}\right\}, \\
w_{\mathrm{i}}=\varphi_{\mathrm{e}}-\pi+i \operatorname{arcosh}\left(k_{x}^{\prime} / k_{x y}\right) .
\end{gathered}
$$

The integration path $C$ in (4) consists of two parts: $C_{+}$, in the half-plane $\operatorname{Im} w>0$, and $C_{-}$, symmetrical to $C_{+}$with respect to the point $w=0$. The path $C_{+}$goes along the parts of three straight lines, from $\varepsilon^{\prime}+i \infty$ to $-\pi-\varepsilon^{\prime}+i \infty$, passing through the points $\varepsilon^{\prime}+i \varepsilon^{\prime \prime}$ and $-\pi-\varepsilon^{\prime}+i \varepsilon^{\prime \prime}$, consecutively, where $\varepsilon^{\prime}$ and $\varepsilon^{\prime \prime}$ should obey the inequalities $0<\varepsilon^{\prime}<\pi / 2$ and $\varepsilon^{\prime \prime}>\left|\operatorname{Im} w_{\mathrm{i}}\right|$. The form of (4), in particular, ensure, that $\left[\rho f_{z}(\chi, \rho, \varphi)\right]^{-1} \partial_{\varphi} f_{z}(\chi, \rho, \varphi)= \pm i k_{x y} \sin \chi$, for $\varphi= \pm \Phi$, and so, the quantity $\chi$ should be taken from (2). The quantity $\operatorname{Re} w_{\mathrm{i}}$ corresponds to the angle (with respect to $x^{\prime}$ axis) of the wave incidence direction. The function $s_{0}(w)$ has the pole at $w=w_{\mathrm{i}}$, corresponding to the incident wave. Also, it can have the poles at $w=2 \Phi-w_{\mathrm{i}}$ (if $\left.\operatorname{Re} w_{\mathrm{i}} \in(\Phi-\pi, \Phi)\right)$ and at $w=-2 \Phi-w_{\mathrm{i}}$ (if
$\left.\operatorname{Re} w_{\mathrm{i}} \in(-\Phi, \pi-\Phi)\right)$, corresponding to the waves reflected from top and bottom faces.

\section{MOVING PARTICLE FIELD}

To use the relations written above for the considered problem solving, one has to present the field connected with the moving charge $e_{0}$ by integral Fourier over the plane waves coming to the line $y^{\prime}=0$ in $2 \mathrm{D}$ problem. The time-dependent Liénard-Wiechert potentials are: $\varphi_{t}^{\mathrm{e}}=e_{0}\left[(\zeta-\beta c t)^{2}+\left(1-\beta^{2}\right)\left(\xi^{2}+\eta^{2}\right)\right]^{-1 / 2}, \quad \vec{A}_{t}^{\mathrm{e}}=\beta \varphi_{t}^{\mathrm{e}} \vec{e}_{\zeta}$. For $\{k>0, \xi>0\}$, performing the Fourier transform, $\mathscr{E}^{\mathrm{e}}=(2 \pi)^{-1} \int_{-\infty}^{+\infty} d t \exp (i k c t) \varphi_{t}^{\mathrm{e}}$, one gets

$$
\begin{gathered}
\mathscr{\phi}^{\mathrm{e}}=e_{0}(\pi \beta c)^{-1} \mathrm{~K}_{0}\left(\kappa_{\xi \eta} \sigma\right) \exp \left(i k_{\zeta} \zeta\right)= \\
=\int_{-\infty}^{+\infty} d k_{\eta} \exp \left(i k_{\eta} \eta+i k_{\zeta} \zeta-\kappa_{\xi} \xi\right) \hat{\varphi}^{\mathrm{e}},
\end{gathered}
$$

where $\hat{\varphi}^{\mathrm{e}}=e_{0}\left(2 \pi \beta c \kappa_{\xi}\right)^{-1}, \sigma=\left(\xi^{2}+\eta^{2}\right)^{1 / 2}, \kappa_{\xi \eta}=k_{\zeta} / \gamma$,

$$
\gamma=\left(1-\beta^{2}\right)^{-1 / 2}, k_{\zeta}=k / \beta, \kappa_{\xi}=\left(\kappa_{\xi \eta}^{2}+k_{\eta}^{2}\right)^{1 / 2},(6)
$$

$\mathrm{K}_{0}$ is McDonald's function. For the relevant transforms of the strength components, denoting $\widehat{E}_{\xi}^{\mathrm{e}}=\kappa_{\xi} \hat{\varphi}^{\mathrm{e}}$, $\widehat{E}_{\eta}^{\mathrm{e}}=-i k_{\eta} \hat{\varphi}^{\mathrm{e}}, \quad \hat{E}_{\zeta}^{\mathrm{e}}=-i k_{\zeta}\left(1-\beta^{2}\right) \hat{\varphi}^{\mathrm{e}}, \quad$ one gets $E_{\xi, \eta, \zeta}^{\mathrm{e}}=\int_{-\infty}^{+\infty} d k_{\eta} \exp \left(i k_{\eta} \eta+i k_{\zeta} \zeta-\kappa_{\xi} \xi\right) \widehat{E}_{\xi, \eta, \zeta}^{\mathrm{e}}\left(k_{\eta}\right)$. For $z-$ components, replacing the integration variable $k_{\eta}$ with $k_{z}, k_{z}=k_{\eta} \sin \theta_{\mathrm{e}}+k_{\zeta} \cos \theta_{\mathrm{e}}$, one comes to the equalities $F_{z}^{\mathrm{e}}=\int_{-\infty}^{+\infty} d k_{z} \exp \left(i k_{z} z+i k_{x}^{\prime} x^{\prime}\right) \tilde{F}_{z}^{\mathrm{e}}\left(k_{z}, \xi\right)$, where

$$
\begin{gathered}
\tilde{E}_{z}^{\mathrm{e}}\left(k_{z}, \xi\right)=i\left(\sin \theta_{\mathrm{e}}\right)^{-1}\left(k \beta \cos \theta_{\mathrm{e}}-k_{z}\right) \hat{\varphi}^{\mathrm{e}} \exp \left(-\kappa_{\xi} \xi\right) \\
\tilde{H}_{z}^{\mathrm{e}}\left(k_{z}, \xi\right)=\beta \kappa_{\xi} \hat{\varphi}^{\mathrm{e}} \exp \left(-\kappa_{\xi} \xi\right) \\
k_{x}^{\prime}=\left(k_{\zeta}-k_{z} \cos \theta_{\mathrm{e}}\right) / \sin \theta_{\mathrm{e}}
\end{gathered}
$$

That is, for the given $k_{z}$, the wedge is illuminated by one E-wave and one $\mathrm{H}$-wave with the amplitudes $\tilde{E}_{z}^{\mathrm{e}}\left(k_{z}, \xi_{\mathrm{e}}\right)$ and $\tilde{H}_{z}^{\mathrm{e}}\left(k_{z}, \xi_{\mathrm{e}}\right)$, respectively. If $k_{z}$ is real and $k_{x}^{\prime}$ is given by (7) then $\left(k_{z} / k\right)^{2}+\left(k_{x}^{\prime} / k\right)^{2} \geq \beta^{-2}>1$, so that these plane waves decrease exponentially in the direction $y^{\prime}$ (same as direction $\xi$ ). The amplitudes depend on $\xi_{\mathrm{e}}$ through the factor $\exp \left(-\kappa_{\xi} \xi_{\mathrm{e}}\right)$, where $\kappa_{\xi}$ is defined in (6) with $k_{\eta}=\left(k_{z}-k_{\zeta} \cos \theta_{\mathrm{e}}\right) / \sin \theta_{\mathrm{e}}$.

\section{DIFFRACTION RADIATION FIELD}

The longitudinal field strength components for the sum of the incident and scattered waves are given by 2D distributions $\tilde{F}_{z}^{\mathrm{e}}\left(k_{z}, \xi_{\mathrm{e}}\right) f_{z}\left(\chi_{F}, \rho, \varphi\right)$. Due to the problem linearity, the $2 \mathrm{D}$ distribution of the scattered field depends on $\xi_{\mathrm{e}}$ through the factor $\exp \left(-\kappa_{\xi} \xi_{\mathrm{e}}\right)$, which is independent on $\rho$ and $\varphi$.

The 3D distributions for the set of waves are given by the equality $\mathbb{F}_{z}(r, \theta, \varphi)=\int_{-\infty}^{+\infty} d k_{z} \exp \left(i k_{z} z\right) F_{z}\left(k_{z}\right)$, where $F_{z}\left(k_{z}\right)=\tilde{F}_{z}^{\mathrm{e}}\left(k_{z}, \xi_{\mathrm{e}}\right) f_{z}\left(\chi_{F}, \rho, \varphi\right)$, and the quantities $\chi_{E}, \chi_{H}$, and $w_{\mathrm{i}}$ are dependent on the ratio $c_{z}=k_{z} / k$ (through (3), (5), and (7)). 
In the wave zone of 2D space (where $k_{x y} \rho>>1$ ) the angular distribution of amplitude is obtained in [5], by the stationary phase method: the contribution of the points $-\pi$ and $\pi$ to the integral in (4) gives

$$
f_{z}\left(\chi_{F}, \rho, \varphi\right) \approx\left[i /\left(2 \pi k_{x y} \rho\right)\right]^{1 / 2} \exp \left(i k_{x y} \rho\right) \Delta_{F}\left(c_{z}, \varphi\right),
$$
where $\Delta_{F}\left(c_{z}, \varphi\right)=\sum_{\{ \pm\}}\left[ \pm s_{z}\left(\chi_{F}, \varphi \mp \pi\right)\right]$ (the dependence on $c_{z}$ is determined through (3), (5), and (7)). The incident and reflected waves related to the uniform charge motion are exponentially decreased in relevant directions, and they do not contribute to the radiation field.

By the same method, in the wave zone of 3D space, where $k r>>1$, one can get the relation

$$
\xi_{z}(r, \theta, \varphi) \approx \exp (i k r) r^{-1} \tilde{F}_{z}^{\mathrm{e}}\left(k \cos \theta, \xi_{\mathrm{e}}\right) \Delta_{F}(\cos \theta, \varphi) \text {. }
$$

For the components of $\mathrm{E}$ - and $\mathrm{H}$-waves, respectively, there are the relations $-E_{z} H_{\varphi}^{*} \approx\left|E_{z}\right|^{2} k / k_{x y}$ and $E_{\varphi} H_{z}^{*} \approx\left|H_{z}\right|^{2} k / k_{x y}$, with asterisk denoting the complex conjugate. The substitution $k_{z}=k \cos \theta$, corresponding to the arguments of the functions in (8), implies $c_{z}=\cos \theta$,

$$
\kappa_{\xi}=k_{\zeta} D^{1 / 2}(\theta) / \sin \theta_{\mathrm{e}}, w_{\mathrm{i}}=\varphi_{\mathrm{e}}-\pi+i \Lambda(\theta),
$$

where $\Lambda(\theta)=\operatorname{arcosh}\left[\left(1-\beta \cos \theta \cos \theta_{\mathrm{e}}\right) /\left(\beta \sin \theta \sin \theta_{\mathrm{e}}\right)\right]$, $D(\theta)=\left(1-\beta^{2}\right) \sin ^{2} \theta_{\mathrm{e}}+\left(\beta \cos \theta-\cos \theta_{\mathrm{e}}\right)^{2}$. The radiation field distribution depends on $\xi_{\mathrm{e}}$ through the factor $\exp \left[-k_{\zeta} \xi_{\mathrm{e}} D^{1 / 2}(\theta) / \sin \theta_{\mathrm{e}}\right]$, independent on $\varphi$. As a result, if the particle motion line is translated parallel to itself then relative distribution of the radiation field with respect to azimuth angle $\varphi$, at the given frequency, for the given polar angle $\theta$, is not changed, the power flux density for the different $\varphi$ is changed in accordance with the same factor written just above. Such azimuthal invariance of the radiation field takes place for the arbitrary $z$-uniform structure, but under the condition of the plane existence, with respect to which the structure and the particle motion line are in the different half-spaces. In such conditions the scattered field distribution is fully determined by the field of external source at the mentioned plane, and the Fourier transforms of the external field components depend on $\xi_{\mathrm{e}}$ exponentially, with increment dependent on $k_{z}$.

Proceeding from the equality

$$
W_{t}=(4 \pi)^{-1} c \int_{-\Phi}^{+\Phi} d \varphi \rho \int_{-\infty}^{+\infty} d z\left(\left[\vec{E}_{t} \vec{H}_{t}\right] \vec{e}_{\rho}\right),
$$

for the power flux through the cylinder with radius $\rho$, performing integration by time, transition to Fourier transforms, the limit transition $\rho \rightarrow \infty$, and denoting

$$
\begin{gathered}
I_{F}(\tau)=\int_{-\Phi}^{+\Phi} d \varphi\left|\Delta_{F}(\tau, \varphi)\right|^{2}, \\
\tilde{W}=(4 \pi)^{-1} c k \int_{-k}^{+k} d k_{z} k_{x y}^{-2} \sum_{F=E, H}\left|F_{z}\left(k_{z}\right)\right|^{2} I_{F}\left(c_{z}\right),
\end{gathered}
$$

for the total radiated energy $W$ one gets

$$
W=4 \pi c \int_{0}^{\infty} d k \tilde{W} .
$$

Replacing of integration variable, $k_{z}=k \cos \vartheta$, gives

$$
\begin{gathered}
\tilde{W}=\left(16 \pi^{3} c\right)^{-1} e_{0}^{2} \int_{0}^{\pi} d \vartheta(\sin \vartheta)^{-1} \exp \left(-2 \kappa_{\xi} \xi_{\mathrm{e}}\right) \times \\
\times\left[I_{H}(\cos \vartheta)+I_{E}(\cos \vartheta)\left(\cos \vartheta-\beta \cos \theta_{\mathrm{e}}\right)^{2} / D(\vartheta)\right] .
\end{gathered}
$$

\section{PERFECTLY CONDUCTIVE WEDGE}

In the case of perfect conductivity, $\operatorname{Im} \chi_{E}=+\infty$, $\chi_{H}=0$, one gets $s_{z}\left(\chi_{E}, \varphi\right)=s_{0}(\varphi), s_{z}\left(\chi_{H}, \varphi\right)=s_{1}(\varphi)$, where $s_{1}(\varphi)=k_{\Phi} \cos \left(k_{\Phi} \varphi\right) /\left[\sin \left(k_{\Phi} \varphi\right)-\sin \left(k_{\Phi} w_{\mathrm{i}}\right)\right]$. In (9), the integrand is rational function of the variable $\exp \left(i k_{\Phi} \varphi\right)$, and the integration gives

$$
\begin{gathered}
I_{E, H}(\cos \vartheta)=2 \pi k_{\Phi} \operatorname{coth}\left[k_{\Phi} \Lambda(\vartheta)\right] \times \\
\times \sin ^{2}\left(\pi k_{\Phi}\right) /\left\{\sinh ^{2}\left[k_{\Phi} \Lambda(\vartheta)\right]+\sin ^{2}\left(\pi k_{\Phi}\right)\right\} .
\end{gathered}
$$

As it follows from (10), (11), and (12), in the case of the perfect conductivity, the total radiated energy does not depend on the angle $\varphi_{\mathrm{e}}$. As a result, if the particle motion line and the edge of wedge are fixed and the wedge is rotated around the edge then the total radiated energy is not changed.

After the next limit transition, to the perfectly conductive half-plane, for which $\Phi=\pi$, from (10), (11), and (12), changing the order of integration, one gets

$$
\begin{gathered}
I_{E, H}(\cos \vartheta)=2 \pi \beta \sin \vartheta \sin \theta_{\mathrm{e}} / D^{1 / 2}(\vartheta), \\
W=3 e_{0}^{2} \gamma \beta^{2} \sin ^{2} \theta_{\mathrm{e}} /\left[8 \xi_{\mathrm{e}}\left(1-\beta^{2} \cos ^{2} \theta_{\mathrm{e}}\right)\right],
\end{gathered}
$$

in agreement with [2] and [3].

Invariance of the radiated energy with respect to the mentioned wedge rotation is connected with the possibility to continue the full field, $\vec{F}^{\mathrm{f}}$, to the space unlimited at an angle $\varphi$, with aid of mirror reflection in the wedge faces, correspondingly to relevant boundary condition. Somewhat similar invariance takes place for the field from a set of point radiation sources. Let, in two 2D problems with the same wave number $k_{x y}$, the external source is given by the same distribution $g^{\mathrm{e}}(\rho, \varphi)$, but shifted at different angles, $g_{j}^{\mathrm{e}}(\rho, \varphi)=g^{\mathrm{e}}\left(\rho, \varphi-\varphi_{j}\right) \quad(j=1,2)$, with all points, in which the Helmholtz equation is not held, $\nabla_{x y}^{2} g_{j}^{\mathrm{e}}(\rho, \varphi)+k_{x y}^{2} g_{j}^{\mathrm{e}}(\rho, \varphi) \neq 0$, remaining in the main sector, $\varphi \in(-\Phi, \Phi)$, for both $j$ values. For the relevant full field, $g_{F j}^{\mathrm{f}}=g_{F j}^{\mathrm{s}}+g_{F j}^{\mathrm{e}}$, the boundary conditions have the form $g_{E j}^{\mathrm{f}}(\rho, \pm \Phi)=0, \partial_{\varphi} g_{H j}^{\mathrm{f}}(\rho, \varphi)_{\varphi= \pm \Phi}=0$.

Let the functions $g_{E j}^{\mathrm{f}}(\rho, \varphi)$ and $g_{H j}^{\mathrm{f}}(\rho, \varphi)$ be continued out of the main sector, $(-\Phi, \Phi)$, with keeping of the equalities $g_{E j}^{\mathrm{f}}(\rho, \pm \Phi+\phi)=-g_{E j}^{\mathrm{f}}(\rho, \pm \Phi-\phi)$ and $g_{H j}^{\mathrm{f}}(\rho, \pm \Phi+\phi)=+g_{H j}^{\mathrm{f}}(\rho, \pm \Phi-\phi)$. The obtained field is $4 \Phi$-periodical in the space unlimited at an angle $\varphi$ and it obeys the Helmholtz equation there, except of the points, which are the mirror reflections of the external source. For the functions $g_{j}^{ \pm}(\rho, \varphi)=\left[g_{E j}^{\mathrm{f}}(\rho, \varphi) \pm g_{H j}^{\mathrm{f}}(\rho, \varphi)\right] / 2$, in the sectors $\varphi-(4 n+1 \pm 1) \Phi \in(-\Phi, \Phi)$, respectively, for any integer $n$, there are no such points. The power radiated in the main sector is half of one radiated in the period.

Change of location of the sources in the main sector by simple translation with respect to angle $\varphi$ gives similar translation of the sources related to the functions $g_{j}^{ \pm}(\rho, \varphi)$ in the space unlimited at an angle $\varphi$, without change of radiation powers associated with these 
sources. The functions $g_{E j}^{\mathrm{f}}(\rho, \varphi)$ and $g_{H j}^{\mathrm{f}}(\rho, \varphi)$, for the given $j$, have opposite parity with respect to mirror reflection in faces, and relevant products give zero contribution to radiation power in one period by angle. As a result, the radiation powers per period for the distributions $g_{j}^{ \pm}(\rho, \varphi)$ have some value $P_{0}$, the same for all four of them, and it is connected with the values $P_{F j}$ of such powers for the distributions $g_{F j}^{\mathrm{f}}(\rho, \varphi)$ by the equality $4 P_{0}=P_{E j}+P_{H j}$. And if the quantities $c_{E j}=P_{E j} /\left(P_{E j}+P_{H j}\right)$ (connected with the ratios of the powers radiated with $\mathrm{E}$ - and $\mathrm{H}$-waves) are identical for both locations of sources, $c_{E 1}=c_{E 2}$, then the powers $P_{E j}$ and $P_{H j}$ also are identical for the different $j$. In particular, in the case of the set of sources corresponding to the uniform charge motion, one has $c_{E}=1 / 2$, $P_{E}=P_{H}=2 P_{0}$, at arbitrary $\varphi_{\mathrm{e}}$ value.

The equality $P_{E}=P_{H}$, taking place for the incident wave

$$
f_{0}^{\mathrm{e}}(\rho, \varphi)=\exp \left[-i k_{x y} \rho \cos \left(\varphi-w_{\mathrm{i}}\right)\right],
$$

is connected with some symmetry between the solutions of 2D Helmholtz equation with the Dirichlet and Neumann boundary conditions. To reveal the symmetry more clearly, it is expedient to solve 2D problem with use of the Kontorovich-Lebedev transforms [6], in the form

$$
\begin{aligned}
& \breve{f}(v)=\int_{0}^{\infty} d \rho \rho^{-1} \mathrm{I}_{v}(\kappa \rho) f(\rho), \\
& f(\rho)=(i \pi)^{-1} \int_{-i \infty+0}^{+i \infty+0} d v v \mathrm{~K}_{v}(\kappa \rho) \breve{f}(v),
\end{aligned}
$$

where $\kappa>0, \mathrm{I}_{v}$ is modified Bessel function. The transformation is used below for $\kappa=k_{x y} / i$, with analytical continuation to $i \kappa>0$, in results.

Let $g_{p}^{ \pm}(\rho, \varphi)(p=0.1)$ be the solutions of the following equation and boundary conditions:

$$
\begin{gathered}
\nabla_{x y}^{2} g_{p}^{ \pm}(\rho, \varphi)+k_{x y}^{2} g_{p}^{ \pm}(\rho, \varphi)=0, \\
g_{0}^{ \pm}(\rho, \mp \Phi)=0, \partial_{\varphi} g_{1}^{ \pm}(\rho, \varphi)_{\varphi=\mp \Phi}=0, \\
g_{0}^{ \pm}(\rho, \pm \Phi)+f_{0}^{ \pm}(\rho)=0, \partial_{\varphi} g_{1}^{ \pm}(\rho, \varphi)_{\varphi= \pm \Phi}+f_{1}^{ \pm}(\rho)=0 .
\end{gathered}
$$

Here $f_{p}^{ \pm}(\rho)=f_{p}^{\mathrm{e}}(\rho, \pm \Phi), f_{1}^{\mathrm{e}}(\rho, \varphi)=\partial_{\varphi} f_{0}^{\mathrm{e}}(\rho, \varphi)$. The transforms $\breve{f}_{p}^{ \pm}(v)$ for the functions $f_{p}^{ \pm}(\rho)$ may be obtained with use of representation of the Bessel function by the Sommerfeld integral [7], in the form

$$
2 \pi \mathrm{I}_{v}(\kappa)=\int d \xi \exp (\kappa \cos \xi-i v \xi)(\operatorname{Re} \kappa>0),
$$

where the integration path goes from $-i \infty-\pi$ to $-i \infty+\pi$ through the points $-\pi$ and $\pi$. With change of integration order, by $\rho$ in (14) and $\xi$ in (17), for $\operatorname{Re} v>0$, one gets

$\breve{f}_{0}^{ \pm}(v)=\exp \left[i v w_{\mathrm{i}} \pm i v(\pi-\Phi)\right] / v, \breve{f}_{1}^{ \pm}(v)=-i v \breve{f}_{0}^{ \pm}(v)$.

If the functions $\breve{g}_{p}^{ \pm}(v, \varphi)$ are taken as following,

$$
\begin{aligned}
& \breve{g}_{0}^{ \pm}(v, \varphi)=-\breve{f}_{0}^{ \pm}(v) \sin (\nu \Phi \pm v \varphi) / \sin (2 v \Phi), \\
& \breve{g}_{1}^{ \pm}(v, \varphi)=\mp i \breve{f}_{0}^{ \pm}(v) \cos (\nu \Phi \pm v \varphi) / \sin (2 \nu \Phi),
\end{aligned}
$$

and the functions $g_{p}^{ \pm}(\rho, \varphi)(p=0,1)$ are determined by them through (15), with $k_{x y} / i$ instead of $\kappa$, then the equation and boundary conditions (16) are held. And then, for the functions $g_{p}^{\mathrm{s}}(\rho, \varphi)=g_{p}^{+}(\rho, \varphi)+g_{p}^{-}(\rho, \varphi)$, the boundary conditions $g_{0}^{\mathrm{s}}(\rho, \pm \Phi)+f_{0}^{\mathrm{e}}(\rho, \pm \Phi)=0$ and $\partial_{\varphi}\left[g_{1}^{\mathrm{s}}(\rho, \varphi)+f_{0}^{\mathrm{e}}(\rho, \varphi)\right]_{\varphi= \pm \Phi}=0$ are held, just as it should be for the scattered field caused by the incident wave (13) and relevant boundary condition. Moving the integration path in (15) to the half-plane $\operatorname{Re} v>0$ and taking the residues in poles, one gets

$$
g_{p}^{\mathrm{s}}(\rho, \varphi)=\sum_{n=1}^{\infty}(-1)^{n} B_{p}\left(n k_{\Phi}, \rho, \varphi\right),
$$

where $\quad B_{p}(v, \rho, \varphi)=B^{-}(v, \rho, \varphi)+(-1)^{p} B^{+}(v, \rho, \varphi)$, $B^{ \pm}(v, \rho, \varphi)=\mathrm{K}_{v}\left(-i k_{x y} \rho\right) \exp \left[i v\left(w_{\mathrm{i}} \pm \varphi\right)\right] s^{ \pm}(v) / \Phi$, $s^{ \pm}(v)=\sin [v \Phi \pm v(\pi-\Phi)]$. In connection with the equality $B^{ \pm}\left(n k_{\Phi}, \rho, \varphi+2 \Phi\right)=(-1)^{n} B^{ \pm}\left(n k_{\Phi}, \rho, \varphi\right)$, the power calculated for the angle interval $\varphi \in(-\Phi, \Phi)$ is half of one calculated for the interval $\varphi \in(-2 \Phi, 2 \Phi)$, the power is given by the sum of contributions of different summands in (18), and the power values in the cases of Dirichlet and Neumann boundary conditions are equal.

\section{RADIATED ENERGY AND IMPEDANCE}

In the Fig. 2, the examples of directional radiation patterns are presented by the quantity $\bar{w}=c \exp \left(2 \kappa_{\xi} \xi_{\mathrm{e}}\right) d W /\left(e_{0}^{2} d \omega \sin \theta d \theta d \varphi\right)$ for the case $\beta=0.9, \alpha_{\mathrm{s}}=45^{\circ}, \Phi=150^{\circ}, \varphi_{\mathrm{e}}=45^{\circ}, \theta_{\mathrm{e}}=60^{\circ}$, at $\theta=30^{\circ}$ (see Fig. 2,a) and $\theta=60^{\circ}$ (see Fig. 2,b). Sloped straight lines correspond to the angles $\pm \Phi$ and $\varphi_{\mathrm{e}}$. The curves correspond to the frequencies, at which $|\mathrm{Z}|=0,0.03$, 0.3 . At the angles $\varphi$ near to 0 and $\pm \Phi$, in both figures, the curves with larger $|Z|$ values give smaller $\bar{w}$ values. At the angles between 45 and $90^{\circ}$, in the Fig. 2,b, on the contrary, the curves with larger $|Z|$ give larger $\bar{w}$.

Appearance of nonzero impedance $\mathrm{Z}$ with $\alpha_{\mathrm{s}} \in(0, \pi / 2)$, can lead both to decrease of the total radiated energy and to its increase. The radiation field is determined by the charge and current distributions on the wedge faces. The increase of impedance corresponds to the resistance increase and can lead to decrease of the currents, and so, to the radiated power decrease. On the other hand, near the surface with nonzero impedance, the surface wave exists (Zenneck's wave) [8]. At $|Z|<<1$, its speed is near to the speed of light. When a particle moves to the edge almost parallel to a face of wedge then it effectively generates the waves, which speed projection on the particle motion direction is near to the particle speed. At the edge of wedge, a part of wave field transforms into a radiation field. In some conditions, an appearance and increase of impedance can yield an increase of the radiated energy. 

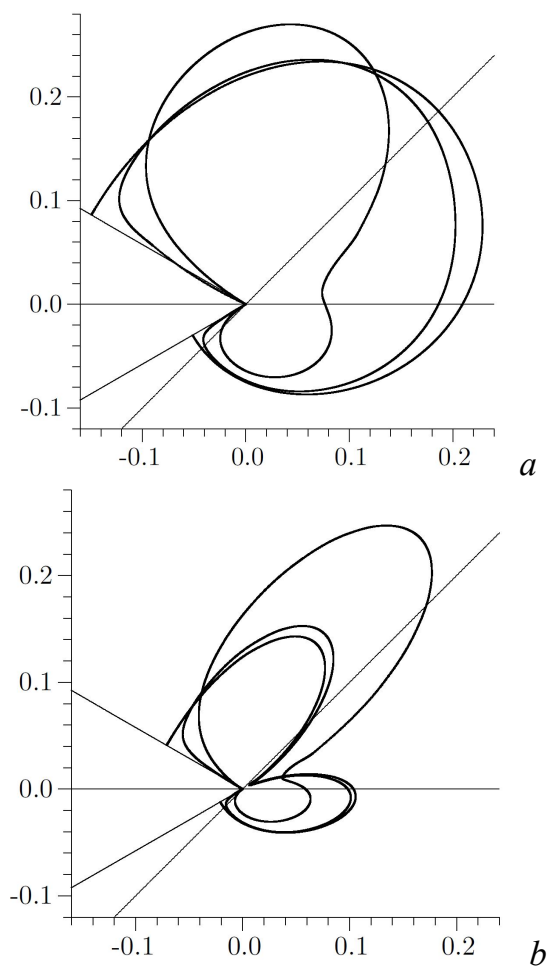

Fig. 2. Directional radiation patterns

To obtain simple approximate relations, it is expedient to consider the case when particle is relativistic, $\gamma \gg 1$, the distance between the motion line and edge is so large, that $\xi_{\mathrm{e}} \gg \gamma \gamma \delta_{\mathrm{s}}$, the value of $\sin \theta_{\mathrm{e}}$ is not small, and the motion line is almost parallel to the lower wedge face, so that the angle $\varphi_{\mathrm{f}}=\varphi_{\mathrm{e}}-\pi+\Phi$ is small, $\varphi_{\mathrm{f}} \ll<1$. Then the main part of energy is radiated within the angle $1 / \gamma$ near the motion directions of the particle and its mirror reflection in the lower wedge face, and for $\left|\theta-\theta_{\mathrm{e}}\right|<<1$ one gets the relations $\Lambda(\theta) \approx\left(1+\tau^{2}\right)^{1 / 2} /\left(\gamma \sin \theta_{\mathrm{e}}\right), \quad D(\theta) \approx \sin ^{2} \theta_{\mathrm{e}}\left(1+\tau^{2}\right) / \gamma^{2}$, $\kappa_{\xi} \approx k\left(1+\tau^{2}\right)^{1 / 2} / \gamma$, where $\tau=\gamma\left(\theta-\theta_{\mathrm{e}}\right)$. Due to the relation $\xi_{\mathrm{e}} \gg \gamma \delta_{\mathrm{s}}$ the factor $\exp \left(-2 \kappa_{\xi} \xi_{\mathrm{e}}\right)$ in (11) may be not small only at the frequencies, which obey the relation $k \delta_{\mathrm{s}}<<1$, and so, give $|\mathrm{Z}|<<1$. That is, the relation $\xi_{\mathrm{e}} \gg \gamma \delta_{\mathrm{s}}$ implies that the main contribution to the integral by $k$ in (10) is given by the frequencies, at which impedance is small. With use of the equality $\prod_{\{ \pm\}} \psi_{\Phi}((\pi / 2) \pm w)=\psi_{\Phi}^{2}(\pi / 2) \cos \left(k_{\Phi} w / 2\right) \quad((4.16) \quad$ in [5]), for $\left\{\left|\varphi-\varphi_{\mathrm{e}}\right|<<1,\left|\theta-\theta_{\mathrm{e}}\right|<<1\right\}$ one gets

$$
\begin{gathered}
s_{z}\left(\chi_{H}, \varphi-\pi\right) \approx 2\left[\varphi_{\mathrm{f}}+i \Lambda(\theta)\right] \times \\
\times\left[\varphi_{\mathrm{f}}+\chi_{H}+i \Lambda(\theta)\right]^{-1}\left(\varphi-\pi+\Phi+\chi_{H}\right) \times \\
\times\left\{\left[\varphi-\varphi_{\mathrm{e}}+2 \varphi_{\mathrm{f}}+i \Lambda(\theta)\right]\left[\varphi-\varphi_{\mathrm{e}}-i \Lambda(\theta)\right]\right\}^{-1},
\end{gathered}
$$

and relevant integration gives $I_{E}(\cos \theta) \approx 2 \pi / \Lambda(\theta)$,

$$
\begin{gathered}
I_{H}(\cos \theta) / I_{E}(\cos \theta) \approx 1-2\left[\Lambda(\theta) \operatorname{Im} \chi_{H}+\varphi_{\mathrm{f}} \operatorname{Re} \chi_{H}\right] \times \\
\times\left\{\left(\varphi_{\mathrm{f}}+\operatorname{Re} \chi_{H}\right)^{2}+\left[\Lambda(\theta)+\operatorname{Im} \chi_{H}\right]^{2}\right\}^{-1} .
\end{gathered}
$$

Denoting $\tau_{\mathrm{f}}=\varphi_{\mathrm{f}} \gamma \sin \theta_{\mathrm{e}}, \quad \tau_{Z}=|Z| \gamma \sin ^{2} \theta_{\mathrm{e}}$, using the variable $\tau=\gamma\left(\theta-\theta_{\mathrm{e}}\right)$, and assuming that $\tau_{\mathrm{f}}$ and $\tau_{Z}$ are not very large, from (11) one gets

$$
\begin{gathered}
\tilde{W}-\tilde{W}_{0} \approx\left(4 \pi^{2} c\right)^{-1} e_{0}^{2} \tau_{z} \times \\
\times \int_{-\infty}^{+\infty} d \tau \exp \left[-2 k \xi_{\mathrm{e}}\left(1+\tau^{2}\right)^{1 / 2} / \gamma\right] \times \\
\times \cos \alpha_{\mathrm{s}}\left[\tan \alpha_{\mathrm{s}}-\tau_{\mathrm{f}} /\left(1+\tau^{2}\right)^{1 / 2}\right] / G(\tau),
\end{gathered}
$$

where $G(\tau)=\left[\left(1+\tau^{2}\right)^{1 / 2}-\tau_{Z} \sin \alpha_{\mathrm{s}}\right]^{2}+\left(\tau_{\mathrm{f}}+\tau_{Z} \cos \alpha_{\mathrm{s}}\right)^{2}$ and $\tilde{W}_{0}$ is the value of $\tilde{W}$ in the perfect conductivity case. From (10) and (19), change of integration order, by $\tau$ and $k$, and change of variable, $\tau=\cot \phi$, gives

$$
\begin{array}{r}
W-W_{0} \approx\left(\pi \delta_{\mathrm{s}}\right)^{-1} e_{0}^{2}\left[\left(\gamma \delta_{\mathrm{s}}\right) /\left(2 \xi_{\mathrm{e}}\right)\right]^{\varpi+1} \gamma \sin ^{2} \theta_{\mathrm{e}} \times \\
\times \Gamma(\varpi+1) \cos \alpha_{\mathrm{s}} S\left(\varpi+1, \tau_{\mathrm{f}}^{2}\right)\left[\tan \alpha_{\mathrm{s}}-R\left(\varpi+1, \tau_{\mathrm{f}}\right)\right],
\end{array}
$$

where $W_{0}$ is related to the perfect conductivity case, $\Gamma$ is gamma-function, $S(p, q)=\int_{0}^{\pi} d \phi(\sin \phi)^{p} /\left(1+q \sin ^{2} \phi\right)$, $R(p, \tau)=\tau S\left(p+1, \tau^{2}\right) / S\left(p, \tau^{2}\right)$. If the faces are resistive ( $\left.\delta_{\mathrm{s}}>0, \cos \alpha_{\mathrm{s}}>0\right)$ then the right hand side of (20) may be both positive and negative. In the case of normal skin effect it is zero at $\tau_{\mathrm{f}} \approx 1.194$. For smaller $\varphi_{\mathrm{f}}$ values, it is positive and appearance of resistance leads to increase of the total radiated energy.

Considering another problem, for the same particle motion and the unbounded impedance plane at $\xi=\xi_{\mathrm{e}}$, one can get the equalities for the field strength components in the form of integrals over $k$ and $k_{\eta}$ of the product of the factor $\exp \left[i k_{\zeta} \zeta-\kappa_{\xi}\left(\xi_{\mathrm{e}}-\xi\right)+i k_{\eta} \eta-i k c t\right]$ $\left(\xi<\xi_{\mathrm{e}}\right)$ with amplitudes dependent on $k$ and $k_{\eta}$. For $k>0$, these amplitudes are inversely proportional to the difference $D_{Z}=\kappa_{\xi}-i k Z$ with $\kappa_{\xi}$ defined by (6), since the dispersion equation for Zenneck's wave has the form $D_{Z}=0$. From the relations $\kappa_{\xi}>0, \operatorname{Im} Z<0$, and $\left|D_{Z}\right|^{2}=\left(\kappa_{\xi}+k \operatorname{Im} Z\right)^{2}+(k \operatorname{Re} Z)^{2}$, it follows that for the sufficiently small value of $|Z|$, its increase leads to decrease of $\left|D_{z}\right|$, and, consequently, to increase of the mentioned amplitudes, and to field amplification.

In the case of the impedance wedge and a particle motion to its edge almost parallel to a face, during the motion near the edge, the relevant amplified field transforms into the radiation field, having greater power, than one in the perfectly conductive wedge case.

\section{CONCLUSIONS}

The wide-band electromagnetic pulse may be generated by particle bunch created in the pulse accelerator and passing by antenna. When the bunch is relativistic then the main part of radiation is emitted in the directions near to the motion directions of the bunch and its mirror reflections in the wedge faces. The cases of the wedge-form antenna with zero and nonzero surface impedance and single particle moving uniformly are considered. For the perfectly conductive wedge, it is shown that if the particle motion line and the edge of wedge are fixed and the wedge is rotated around the edge then the total radiated energy is not changed. Appearance and increase of impedance may lead both to decrease and to increase of the total radiated energy. The decrease may be caused by decrease of the surface currents, through the resistance increase. The increase of the radiated en- 
ergy takes place in the conditions, favorable for the surface wave generation, when the particle moves to the edge almost parallel to a face of wedge. Both for perfectly conductive wedge and for impedance one, at the given frequency, the relative distribution of the radiation field in directions that form a fixed angle with the edge of wedge is not changed if the particle motion line is translated parallel to itself. Such invariance of the radiation field takes place for the arbitrary structure uniform in some direction, under the condition of the plane existence, with respect to which the structure and the particle motion line are in the different half-spaces.

\section{REFERENCES}

1. V.A. Balakirev, N.I. Gaponenko, A.M. Gorban', et al. Excitement of TEM-horn antenna by impulsive relativistic electron beam // Problems of Atomic Science and Technology. Series "Plasma Physics". 2000, № 3, p. 118-119.
2. A.P. Kazantsev, G.I. Surdutovich. The radiation of the charged particle flying near the conductive screen // Dokl. Akad. Nauk SSSR. 1962, v. 147, № 1, p. $74-77$ (in Russian).

3. A.P. Potylitsyn, M.I. Ryazanov, M.N. Strikhanov, A.A. Tishchenko. Diffraction radiation of the charged particles. Tomsk, 2008, $347 \mathrm{p}$.

4. G.D. Malyuzhinets. The excitation, reflection and radiation of surface waves in a wedge-like region with given face impedances // Dokl. Akad. Nauk SSSR. 1958, v. 121, № 3, p. 436-439 (in Russian).

5. V.M. Babich, M.A. Lyalinov, V.E. Grikurov. Sommerfeld-Malyuzhinets technique in diffraction theory. Saint-Petersburg, 2004, 103 p.

6. H. Bateman, A. Erdelyi. Tables of integral transforms. V. 2, McGraw-Hill, 1954.

7. H. Bateman, A. Erdelyi. Higher transcendental functions. V. 2. McGraw-Hill, 1953.

8. L.A. Vainstein. Electromagnetic waves. Moscow: "Radio i Sviaz", 1988, 440 p. (in Russian).

Article received 04.01.2021

\section{ДИФРАКЦИОННОЕ ИЗЛУЧЕНИЕ ПРИ ДВИЖЕНИИ ЧАСТИЦЫ МИМО ИМПЕДАНСНОГО КЛИНА}

\section{В.М. Остроуико}

Рассмотрено поле дифракционного излучения, созданного заряженной частицей при движении мимо идеально проводящего или импедансного клина. При идеальной проводимости поворот клина вокруг ребра, фиксированного вместе с линией движения частицы, не изменяет полной излученной энергии. При движении частицы к ребру почти параллельно к грани клина увеличение импеданса от нуля увеличивает упомянутую энергию.

\section{ДИФРАКЦЙНЕ ВИПРОМІНЮВАННЯ ПРИ РУСІ ЧАСТИНКИ ПОВЗ ІМПЕДАНСНИЙ КЛИН}

\section{В.М. Остроушко}

Розглянуте поле дифракційного випромінювання, утвореного зарядженою частинкою при русі повз ідеально провідний або імпедансний клин. При ідеальній провідності обертання клину навколо ребра, фіксованого разом з лінією руху частинки, не змінює загальної випроміненої енергії. При русі частинки до ребра майже паралельно до грані клину, збільшення імпедансу від нуля збільшує згадану енергію. 